\title{
Grooving Phenomenon Analysis of Series-Connected Augmented Sextupole Rails Material
}

\author{
Xinpeng XUE, Tao SHU, Ming LIU, Zhiyong YANG \\ Air Force Engineering University \\ Xi'an, China \\ e-mail: xue_xinpeng0708@163.com
}

\begin{abstract}
Because the electromagnetic railgun's main current can reach several MA and the muzzle velocity is more than 2 $\mathbf{k m} / \mathrm{s}$, launching in this harsh environment in barrel will cause grooving to the rail. Using existing experimental data of references, this paper analyses the position and depth analysis of the grooving phenomenon. So this paper design series connected augmented sextupole rails electromagnetic launcher to obtain high inductance gradient. High inductance gradient plays an important role in a novel electromagnetic launcher. Since inductance gradient is the decisive factor to super high speed and reduce high current, this study focuses on the design of launch model and inductance gradient distribution in moving area of double projectile. In this study we will simulate three typical series augmented sextupole rails connection mode, with finite element method and compare them. The theoretical analysis and numerical simulations indicate that the inductance gradient performance of the A configuration is better than other configurations. Therefore, this configuration could reduce the grooving phenomenon of sextupole rails.
\end{abstract}

Keywords-large pulse current; grooving phenomenon, series-connected augmented sextupole rails electromagnetic launcher; inductance gradient

\section{INTRODUCTION}

Since the successful launch of multiprojectile using multiturn electromagnetic technology in the 1980s, the world continues a broad and deep research to develop electromagnetic launch technology[1]. Railgun has the potential to accelerate projectile to super high speed. Using high density energy storage system, the pulsed power could provide the current of $6 \mathrm{MA}$ to railgun. However, the high current might reach a danger level that will lead to grooving phenomenon and deterioration of the rails[2].

Based on the background of conventional electromagn-etic launchers don't solve the problem of grooving phenomenon during the launching process, this paper presents a new electromagnetic launcher:series-connected augmented sextupole rails electromagnetic launcher. The use of series-connected augmented sextupole rails can solve the problem of grooving phenomenon of sextupole rails.

\section{GROOVING PHENOMENON OF RAIL MATERIAL}

In 1998, R. M. Gee first discovered grooving phenomenon on the medium calibre launcher of the institute of high technology(IHT)[4], when the track is Be-Cu, 10 test, track channel erosion damage obviously, contour detection of $\mathrm{Be}-\mathrm{Cu}$ damage of the cross-sectional area, found a groove into the lip on the two side edge of the track; for $\mathrm{W}-\mathrm{Cu}$, $\mathrm{Cr}-\mathrm{Cu}$ and $\mathrm{Ds}-\mathrm{Cu}$ have no grooving phenomenon. At that time, R. M. Gee attributed the grooving phenomenon to plastic deformation of the material, but no other work was done[4].

In 2002, IHT began to study the grooving phenomenon, when the aluminum alloy track and aluminum alloy armature pairing were tested under high current condition. Obvious track damage (especially at the initial stage) appears in the test, which makes people pay more attention to the grooving phenomenon.

\section{A. Position of Grooving Phenomenon}

The position of grooving phenomenon is located on the edge of both sides of the track, corresponding to the armature edge position. In 2006, the analysis of track grooving phenomenon under multiple launch conditions by T. Watt showed that the position of grooving phenomenon was located at the maximum track current and the longest contact time with the armature, that is, the edge of the track near the insulation support[3]. This position corresponds to the most serious damage to the armature[5,6]. At $10 \mathrm{~cm}$ from the end of the gun, the groove and the insulation support edge coincide, and the edge line of the pit is clear. At the distance of $30 \mathrm{~cm}$ from the end of the gun, a part of the groove has been extended into the insulation support area. In 2012, the U.S. Navy laboratory completed the steel rail wear test[2], the most serious grooving phenomenon area is $4 \mathrm{~mm}$ from the edge of the track, which is also corresponding to the armature edge position, and the groove width is about $3-4 \mathrm{~mm}$. The test also shows that the pitting corrosion pit is centered on the edge line of the armature fin, and extends to both sides simultaneously.

In 2006, K. P. Copper carried out multiple cumulative emission tests, measured the distance from the bottom of the groove pit at the depth of $36 \mathrm{~cm}$ and $96 \mathrm{~cm}$ from the firing depth to the distance from the orbital edge[7]. It is found that the positions of slots and pits vary little with the number of launches; however, the groove pits on both sides of the track edge are not completely symmetrical. The distance between the groove along the track and the edge of the track is $3.5 \mathrm{~cm}$ on average, $36 \mathrm{~cm}$ away from the track, and the distance between the groove along the track and the edge of the track is $4.9 \mathrm{~cm}$, while the average distance between the track and the edge of the track at $94 \mathrm{~cm}$ from the end of the gun is $3.2 \mathrm{~cm}$. In the analysis of orbital slot corrosion under multiple launch conditions, T. Watt found that the groove near the upper insulating support is wider than the lower side[3]. 


\section{B. Depth of Grooving Phenomenon}

Depth of grooving phenomenon usually reaches several millimeters, and accumulation effect exists. In 2004, R. A. Meger found that there were obvious wear and melting traces in the track erosion area, and the size was about 150 micron [8]. In the steel track wear test carried out by the Navy Laboratory of the United States, the profile of the cross section at different positions along the trajectory of the track was measured, and the total deformation of the track was taken into account, and the correction was made[2]. The most serious point of corrosion is about 300 micron away from the surface. The multiple accumulated emission test is based on the surface of the center of the track, and the depth of the pitting corrosion is shown in figure 1[7]. The depth of grooving phenomenon has obvious linear relationship with the number of firing.

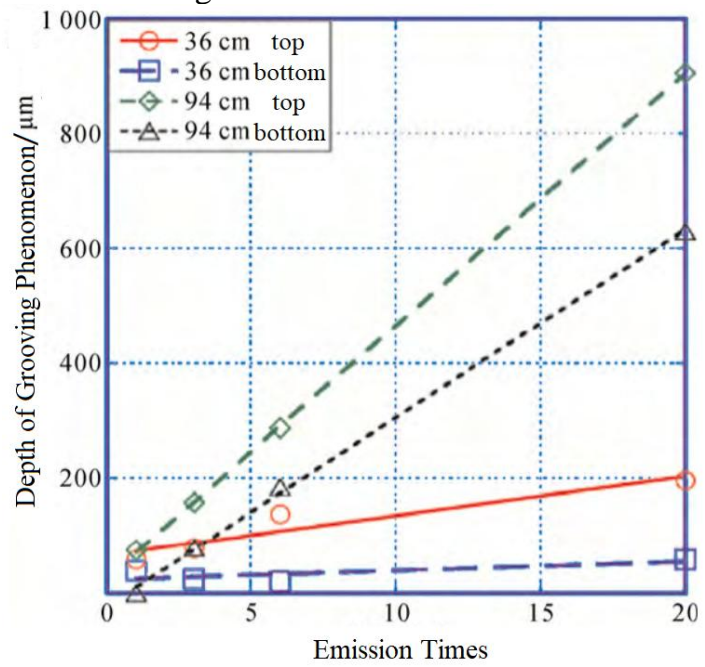

Figure 1. Graph showing plots of groove depth as a function of emission times.

We can see from Fig. 1, the pit breech at $94 \mathrm{~cm}$ than at $36 \mathrm{~cm}$ from the breech of the deep 4-6 times, the track grooving phenomenon along than along the depth of 1.5-2 times; from the groove depth of corrosion pit and launches the breech at $94 \mathrm{~cm}$ completely proportional the relationship between groove depth and pit. There is a non proportional relationship between the depth of crater and the number of firing at the distance of $36 \mathrm{~cm}$ from the breech (about 2 times the depth of groove corrosion corresponding to the firing number is less than 1.33 times).

\section{SEXTUPOLE RAILS TOROIDAL MAGNETIC FIELD CONFIGURATION}

The series-connected augmented sextupole rails electromagnetic launcher with intelligent ammunition is presented in Fig. 2. This launcher mainly consists of series augmented sextupole rails and double-projectile. The material of the rails and projectiles is copper and aluminium. In series-connected augmented electroma- gnetic launcher, it has double-sextupole-rails and two separate projectiles. Two sextupole rails pairs are stacked and each connected with an independent projectile and separated by an insulating space in $\mathrm{Z}$ axis. The structure is symmetric in $\mathrm{X}-\mathrm{Y}$ plate. Similar to practical case, the external devices include a very high and shortly current pulse energy system is applied to the series-connected augmented electromagnetic launcher.

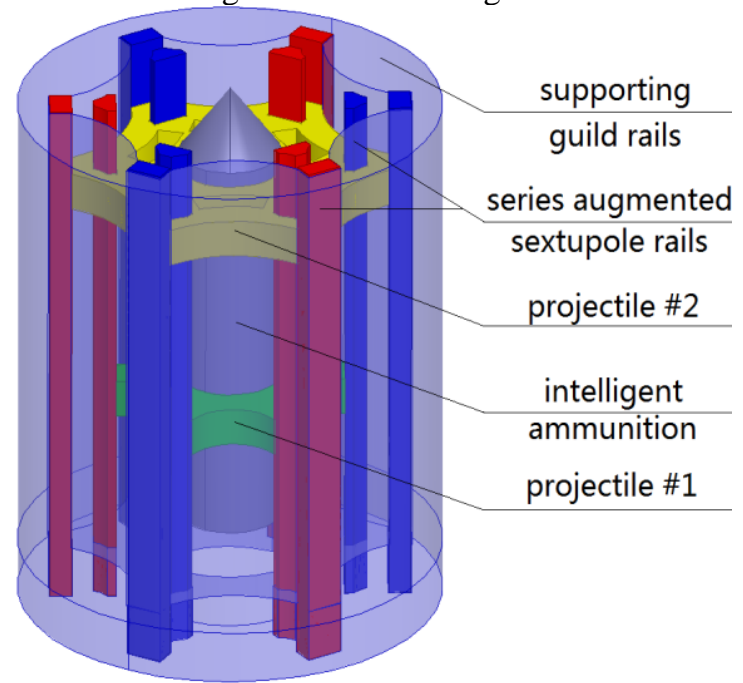

Figure 2. Series-connected augmented sextupole rails electromagnetic launcher model.

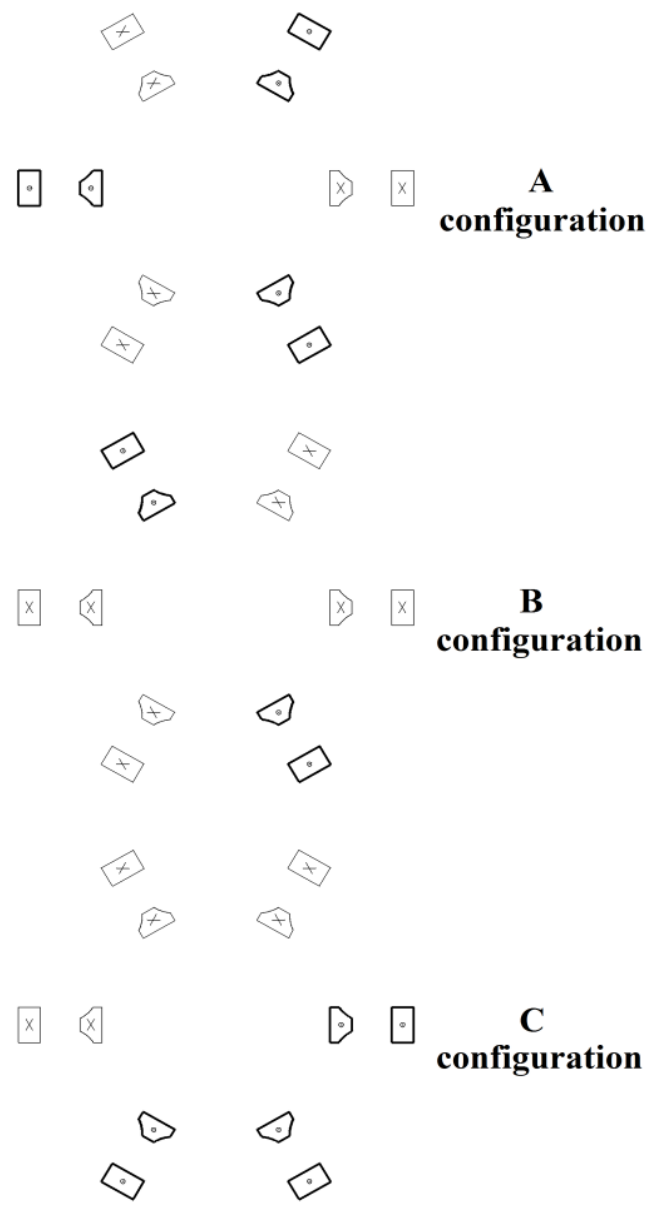

Figure 3. The three typical series augmented sextupole rails connection mode. The current flows out of rail is represented by the bold line. And the current flows into rail is represented by the ceriph. 

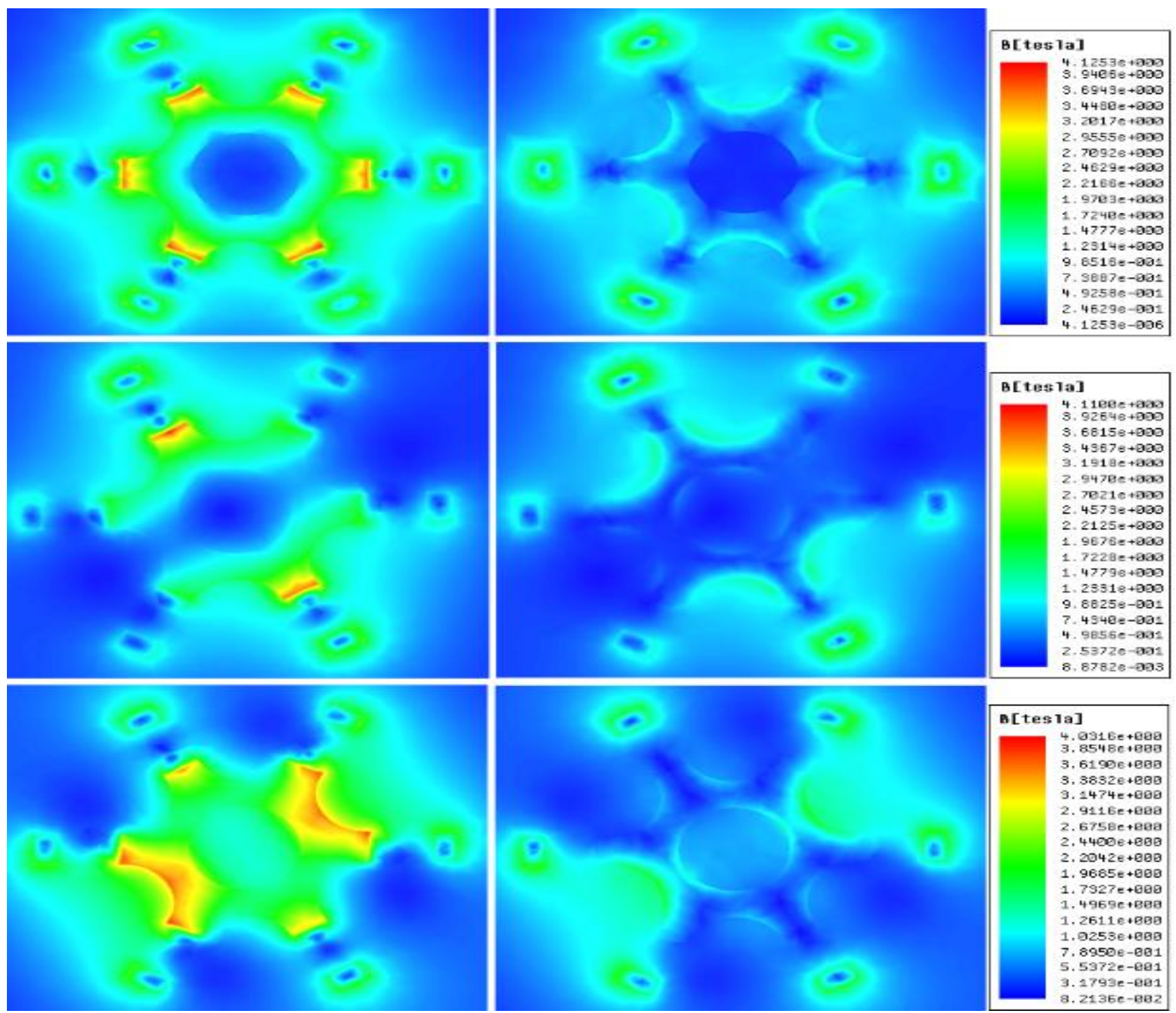

Figure 4. The magnetic field intensity nephogram of threeconfigurations. From top to bottom in order is A, B, C three configurations. Left side for bottom section and right side for upper surface $1 \mathrm{~mm}$ of projectile\#1

\section{A. Typical Configuration}

Structurally, the traditional augmented railguns of launching massive projectile can be regarded as a series augmented dipole rails electromagnetic launcher. And the series augmented sextupole rails can be considered as a superposition of multiple augmented railguns. For the traditional augmented railguns, the currents of two pairs rails must be opposite. The two rails form a current loop through massive projectile and provide the same direction of magnetic field in the motion area. This is the necessary condition of electromagnetic driving force.This restriction allows traditional railguns to have only one kind of magnetic field configuration. While, series augmented sextupole rails electromagnetic launcher can produce a variety of magnetic field configuration by changing the currents direction of each rails.
For the series sextupole rails electromagnetic launcher, Fig. 3 shows the three typical series augmented sextupole rails connection mode. In order to ensure the symmetry of radial force, the configuration of the currents asymmetry is not considered. Meanwhile,for meeting the requirement of the system circuit, six pairs rails can not produce inward or outward currents at the same time. Therefore, only A, B, C three configurations meet this requirement.

\section{B. Results and Analysis of Simulation}

Analysing the distribution of magnetic field, select the bottom section and upper surface $1 \mathrm{~mm}$ of projectile\#1 as the section magnetic field. Fig. 4 shows the magnetic field intensity nephogram of three configurations. In the case of rail section current is 200kA, The maximum magnetic field produced by the three configurations are 4.13T, $4.11 \mathrm{~T}$ and 4.03T. Without exception, all appeared in the contact surface between the sextupole rails and projectile. If the currents 
direction of the adjacent rails is opposite, it can enhance the magnetic field of the rails. This phenomenon can be clearly observed in the magnetic field distribution of $\mathrm{B}$ and $\mathrm{C}$ configurations. Compared with the other two configurations, the magnetic field intensity of the hexagon region surround by A configurations rails is the largest, while, magnetic field is the least in central region. It shows that this configuration can converge magnetic lines of force in the motion of the double-projectile. In addition, contrasting the magnetic field intensity of upper surface $1 \mathrm{~mm}$ of projectile\#1, A configuration decay of the fastest. This shows that the magnetic field changes the most rapidly along the axial direction of the A configuration. Accordingly, the mutual gradient of the sextupole rails and the double-projectile is also largest, all of which benefits to accelerate projectile as well. Inferred from the magnetic field distribution, the acceleration performance of $\mathrm{A}$ configuration is the best and $\mathrm{B}, \mathrm{C}$ are gradually decreased.

From the field lines reconnection point of view, the spacing of two rails should be reduced as much as possible, so that magnetic lines of force is the shortest,and magnetic reluctance is the least. Similarly, in the series augmented sextupole rails configuration, for reducing magnetic reluctance, the direction of the field lines by the adjacent rails should together and pass the middle guide arc. The current of A configuration of each rail and the adjacent rails is reverse, the field lines reconnection most easily achieved. However, the field lines path of $\mathrm{B}$ and $\mathrm{C}$ configuration increases progressively.

\section{NUMERICAL SimUlation EVALUATION}

\section{A. Power Circuit Consideration}

Different from the coilgun or toroidal electromagnetic launcher for strict limits to the initial discharge position [1], the series augmented sextupole rails electromagnetic launcher does not require complex controllers and simplify the complexity of power circuit. The electrical component arrangement of A configuration series augmented sextupole rails electromagnetic launchers was shown as Fig. 5. Energy system used high energy storage capacity to generate large pulse current for discharging to series augmented sextupole rails. $D$ is the continuous current diode. $R(t)$ is the dynamic resistance of the rails circuit. Rail\#11 to rail\#26 are the self-inductance of the series augmented sextupole rails. The function of D11, D13, and D15 is to control the current direction of rail\#11, rail\#13, and rail\#15, respectively. Similarly, the function of D21, D23, and D25 is to control the current direction of rail\#21, rail\#23, and rail\#25.

\section{B. Axial Acceleration Force of Double-Projectile}

The effect thought of axial thrust force is the same, whether it is projectile\#1 or projectile\#2. The series augmented sextupole rails can be considered as many independent linear conductor and produce double multipulse toroidal magnetic field. Since the space relation of series augmented sextupole rails and the shape design of double projectile, the currents of projectile along the toroidal direction. The series augmented sextupole toroidal magnetic field interacts with orthogonal to the field to generate axial ampere-force. Fig. 6 shows the vector of toroidal fields and orthogonal to the field in 3D finite element software.

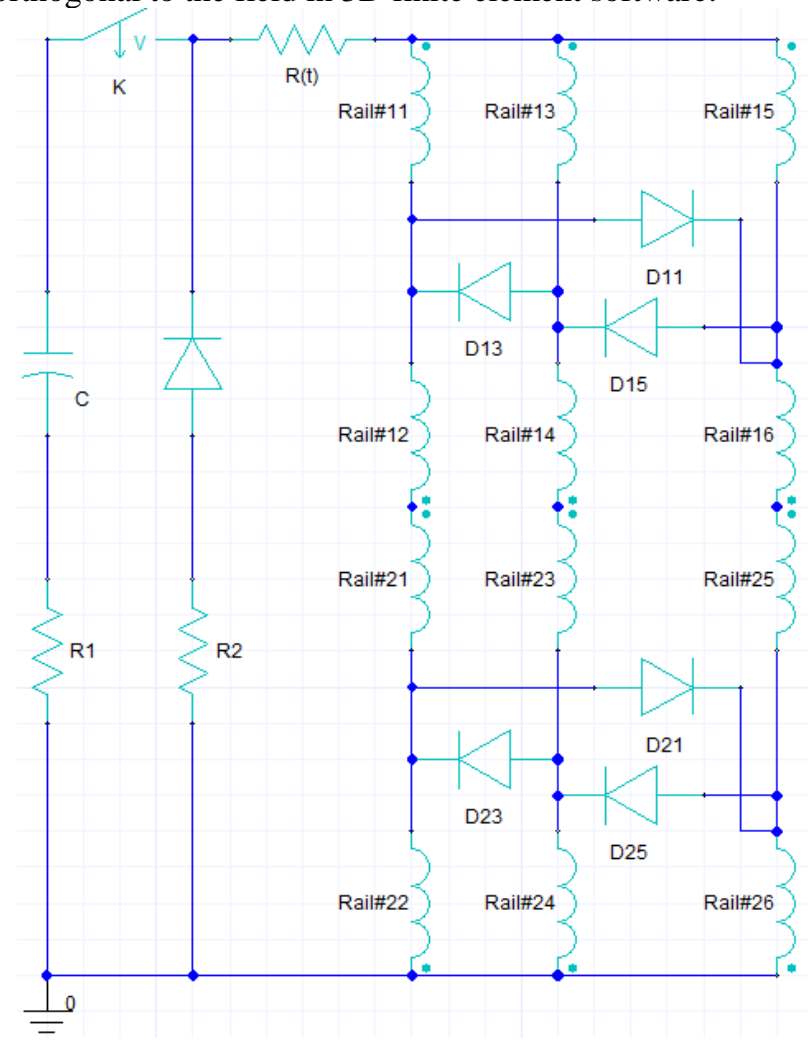

Figure 5. The electrical component arrangement of A configuration series augmented sextupole rails electromagnetic launchers. The rail number starting from 11 means the inner and the first pole rail. Sixtupole rails lined in a clockwise direction.

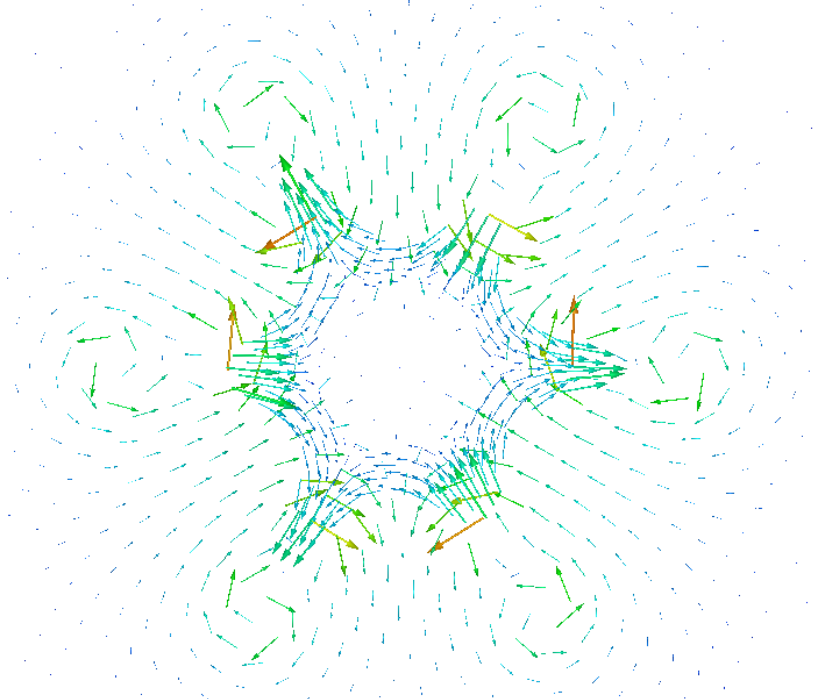

Figure 6. Vector of sextupole toroidal fields and orthogonal to the field The direction of the toroidal currents in the club-shaped projectile were orthogonal to the sextupole toroidal fields everywhere. 


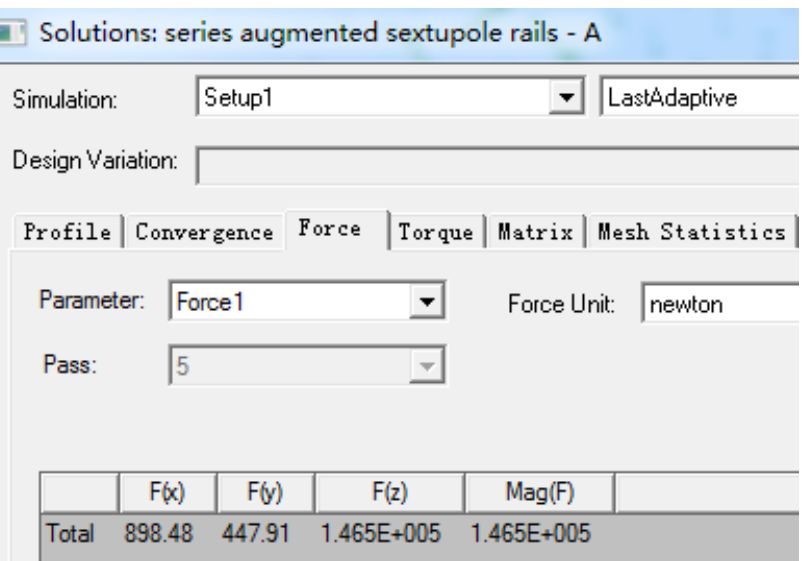

Figure 7. Propulsion force acted on the projectile. The axial propulsion force is $1.465 \times 105 \mathrm{~N}$, however, the interference forces of force $\mathrm{x}$ and force_y are very small comparing with propulsion force.

Owing to the direction of magnetic field and current are perpendicular to each other, the sextupole toroidal magnetic field interacts with the orthogonal to the field and produces huge axial propulsion force. In case of the current of series augmented sextupole rails is $200 \mathrm{kA}$, the propulsion force acted on projectiles was shown in Fig. 7.

Due to the "double projectile" approach, the actual sliding surfaces is increased by six times comparing with the traditional railgun. The friction between each side of theprojectile and rail can be considered as a fixed value, this is true that would increase friction and wear. While, the design of rails by a factor of six not only achieves better thrust/current ratios, but better thrust/friction ratios.

\section{CONCLUSION}

This paper has presented the theoretical analysis and numerical evaluation of the series-connected augmented sextupole rails electromagnetic launcher. The effect thought of electromagnetic force is the toroidal magnetic fields interact with orthogonal to the field to generate axial acceleration force. Numeric simulation results indicate that magnetic shielding effect of the A configuration is better than two other configurations and will help the intelligent ammunition launch. This new design electromagnetic launcher can achieve stated objectives of increasing overall propulsive forces for a given current and decreases fringing magnetic fields. For huge thrust force and launching intelligent ammunition, this design has an obvious advantage.

\section{REFERENCES}

[1] Xinpeng Xue, Tao Shu, Zhiyong Yang, and Gang Feng, "Toroidal Field Electromagnetic Launcher," IEEE Trans. Plasma Science, vol. 44, pp. 2393-2398, September, 2016.

[2] Meger R A, Cairns R, Doulass S, et al. EM gun bore life experiments at the naval research laboratory $[\mathrm{C}] / / 2012$ 16th International Symposium on Electromagnetic Launch Technology. [S.1.]: IEEE, 2012: 1533-1537.

[3] Watt T, Stefani F, Crawford M, et al. Investigation of damage to solid-armature railguns at startup[J]. IEEE Transactions on Magnetics, 2007, 43(1): 214-218.

[4] Gee R M, Persad C. The response of different copper alloys as rail contacts at the breech of an electromagnetic launcher[J]. IEEE Transactions on Magnetics, 2001, 37(1): 263-268.

[5] Stefani F, Merrill R. Experiments to measure melt-wave erosion in railgun armatures[J]. IEEE Transactions on Magnetics, 2003, 39(1):188-192.

[6] Watt T, Stefani F. The effect of current and speed on perimeter erosion in recovered armatures[J]. IEEE Transactions on Magnetics, 2005,41(1): 448-452.

[7] Cooper K P, Jones H, Meger R A. Analysis of railgun barrel materials[J]. IEEE Transactions on Magnetics, 2007, 43(1): 120-125.

[8] Meger R A, Cooper K P, Jones H N, et al. Analysis of rail surfaces from a multishot railgun[J]. IEEE Transactions on Magnetics, 2005,41(1): 211-213. 